(2) Open Access Full Text Article

REVIEW

\title{
Human normal immunoglobulin in the treatment of primary immunodeficiency diseases
}

This article was published in the following Dove Press journal:

Therapeutics and Clinical Risk Management

30 March 2012

Number of times this article has been viewed

\section{Philip Wood \\ St James University Hospital, Leeds, United Kingdom}

Correspondence: Philip Wood St James' University Hospital, Leeds LS9 7TF, United Kingdom Tel +44 II 32067256

Fax +44 II 32067250

Email philipwoodI@nhs.net
Abstract: The primary antibody deficiency syndromes are a rare group of disorders that can present at any age, and for which delay in diagnosis remains common. Replacement therapy with immunoglobulin in primary antibody deficiencies increases life expectancy and reduces the frequency and severity of infection. Higher doses of immunoglobulin are associated with reduced frequency of infection. Late diagnosis and delayed institution of immunoglobulin replacement therapy results in increased morbidity with a wide variety of organ-specific complications and increased mortality. Risks of immunoglobulin therapy are minimized by modern manufacturing processes, although patients can experience both immediate and delayed adverse reactions, and concerns remain over the transmission of prions in plasma. Immunoglobulin therapy leads to improvements in overall quality of life, and many of the improvements relate to reduced infection rates and fear of future infections, strongly suggesting that the immunoglobulin therapy itself is the major factor in this improvement. There are limited data on the economic benefits of immunoglobulin therapy, with the fluctuating costs of immunoglobulins making comparison between different studies difficult. However, estimates suggest that early intervention with immunoglobulin replacement compares favorably with prolonged therapy for other more common chronic diseases.

Keywords: antibody deficiency, immunoglobulin therapy, common variable immunodeficiency

\section{Introduction}

The primary immunodeficiencies are a rare group of disorders in which the fundamental defect is an inability to maintain an effective immune response to an invading pathogen. They can be categorized into a number of different groups:

- Combined immunodeficiencies, in which defects of both cellular and humoral immunity result most commonly from single defects in genes encoding proteins critical for lymphocyte development; these disorders usually present in early childhood and are usually fatal without hematopoietic stem cell transplantation.

- Antibody deficiencies, which result from a variety of single gene defects, and most commonly more complex polygenic disorders, in which failure of an effective immunoglobulin response to infection places the individual at significant risk of life-threatening infection, most commonly with encapsulating bacteria, such as Streptococcus pneumoniae or Haemophilus influenzae.

- Complement deficiencies, in which a genetically defined inability to produce complement components places the individual at risk of a variety of infections and potentially inflammatory complications. 
- Phagocytic disorders, most notably chronic granulomatous disease, usually as a result of X-linked or autosomal recessive inheritance; susceptibility to infection with catalase negative bacteria, most typically Staphylococcus aureus, resulting in abscesses and granuloma formation, is the hallmark of this group of disorders.

- Defects in innate immunity, at present most commonly those involving the Toll-like receptor pathways, where the lack of an effective inflammatory response both increases susceptibility to infection from bacterial and fungal pathogens and results in tissue damage as a consequence of recurrent infection.

Immunoglobulin replacement therapy is most commonly indicated in defects of antibody production, but is often used in the context of severe combined immunodeficiency, both before and after stem cell transplant, where poor B cell engraftment may require long-term immunoglobulin support. Some defects in innate immunity, in particular the hyper $\operatorname{IgE}$ syndrome, can develop a degree of antibody deficiency which may require immunoglobulin replacement therapy.

The remainder of this paper discusses the history of immunoglobulin replacement therapy, current practices, and the products available, focusing on the therapeutic benefit and risk of treatment. The primary antibody deficiencies are focused upon, given that these are the most common of the primary immunodeficiencies, and for which immunoglobulin replacement therapy is most widely indicated. In addition, the evidence of efficacy for immunoglobulin replacement therapy is most clearly demonstrated in this group of disorders.

\section{Clinical presentation}

The International Union of Immunological Societies has developed diagnostic criteria for a wide range of primary immunodeficiencies, including the commoner primary antibody deficiencies. ${ }^{1}$ Infections with encapsulated bacteria such as $H$. influenzae and S. pneumoniae are the commonest presenting features, ${ }^{2,3}$ with recurrent pneumonia, sinusitis, otitis media, and acute bronchitis being most common infective histories obtained from patients presenting with primary antibody deficiency. Infections often respond to standard treatment, only to recur once therapy has finished. Bronchiectasis and chronic sinusitis are common complications before diagnosis and treatment. ${ }^{4}$ Although bacterial infections are the most common, patients with the common variable immunodeficiency spectrum of disorders are prone to fungal, viral, and protozoal infection, including opportunistic organisms, particularly when there is $\mathrm{T}$ lymphopenia or evidence of $\mathrm{T}$ cell dysfunction.
In addition to these infective presentations, underlying dysregulation of the immune system, thought to be inherent in common variable immunodeficiency, is illustrated by the observation that patients can present with systemic or organ-specific autoimmunity., 2,3 This is most commonly hematological. Other organ-specific autoimmunity, eg, pernicious anemia secondary to autoantibodies directed against intrinsic factor, is also common and can be the presenting feature of the condition.

A subgroup of patients with common variable immunodeficiency can present with or develop a granulomatous syndrome affecting the liver, spleen, lungs, and gastrointestinal tract during the course of their disease. This can often appear similar to other granulomatous conditions, such as Crohn's disease or sarcoidosis, and can lead to diagnostic confusion and delay in appropriate therapy.

\section{History of immunoglobulin therapy}

Following the report by Colonel Ogden Bruton in 1953 of what was subsequently identified as X-linked agammaglobulinemia ${ }^{6}$ treated with replacement plasma, early attempts to replace absent immunoglobulin progressed from the use of fresh frozen plasma to relatively impure preparations of immunoglobulin given intramuscularly.

The processes of cold-ethanol and $\mathrm{pH}$ fractionation to extract immunoglobulin from plasma were developed in the $1940 \mathrm{~s}$, with preparations containing $70 \%-80 \%$ monomeric IgG and substantial amounts of IgA and IgM. Such preparations proved useful in reducing infections in patients with X-linked agammaglobulinemia when given intramuscularly, but produced life-threatening anaphylactic reactions when given intravenously. Enzymatic modifications of IgG resulted in more monomeric preparations, but with a significant loss of function, including complement-binding activity.

Identification of processes that could result in the preparation of intact IgG at high purity, involving low $\mathrm{pH}$ and trace pepsin concentrations, precipitation by polyethylene glycol, or purification using diethyldiaminoethyl ionexchange chromatography, paved the way for development of stable products that could be administered intravenously, and many patients with primary antibody deficiencies were moved onto these newer preparations.

\section{Modern manufacturing processes}

The quality of plasma collected directly impacts on the final quality of the intravenous immunoglobulin or subcutaneous immunoglobulin preparation. Strict quality assurance 
measures in place throughout the process ensure high levels of reliability and consistency. Collection centers are overseen by national and international regulatory authorities, and should comply with Good Manufacturing Practice. Plasma donors have a documented medical history and should be exempt from risk factors for plasma-borne infectious agents. Upon collection, most plasma for intravenous immunoglobulin is frozen to $-25^{\circ} \mathrm{C}$ or $-30^{\circ} \mathrm{C}$ within 24 hours, and kept in this state for several months.

Individual donations are screened for human immunodeficiency virus (HIV) 1 and 2 and hepatitis C antibodies, as well as hepatitis B surface antigen. Many manufacturers now screen minipools of donations for genomic viral markers of HIV, hepatitis A, B, and C, and parvovirus B19. The manufacturing pool should then screen negative for the hepatitis $\mathrm{C}$ virus nucleic acid test, HIV antibodies, and hepatitis B surface antigen, often now with additional screening for hepatitis A RNA and parvovirus B19 DNA.

In most processes, plasma is then subjected to controlled thawing at $2^{\circ} \mathrm{C}-3^{\circ} \mathrm{C}$, known as cryoprecipitation, with the cryoprecipitate removed, leaving a "cryo-poor" fraction containing the immunoglobulin, after removal of fibrinogen by ethanol precipitation at neutral $\mathrm{pH}$. Subsequent processes may involve ion-exchange chromatography, use of caprylic acid, incubation at low $\mathrm{pH}$, and nanofiltration to ensure the highest purity and maximal yield.

Previously, the end-products were produced in lyophilized form, but this resulted in a risk of aggregate formation upon reconstitution, and the discovery that $\mathrm{IgG}$ remains stable in liquid form at $\mathrm{pH} 4.25$ and that patients could tolerate such preparations has resulted in a move to liquid preparations at low $\mathrm{pH}$ with the addition of stabilizers, such as polyols, sugars, and, increasingly, amino acids, such as proline or isoleucine.

For a number of years, intravenous immunoglobulin products were provided at 5\% concentration, requiring a number of hours of infusion time and with the disadvantage of comparatively high total volumes. The latter issue could be of relevance in elderly or frail patients with cardiorespiratory disease or renal impairment. More recently, manufacturers have moved to more concentrated products for intravenous use, typically $10 \%$, resulting in faster infusion times and smaller overall volume product. ${ }^{7-10}$

Early subcutaneous immunoglobulin products were developed using products originally designed for intramuscular use, typically at $16 \%$ concentration. More recently, CSL Behring has developed a $20 \%$ product (Hizentra ${ }^{\circledR}$ ), for subcutaneous use. Again, the smaller volumes and higher concentration bring the potential benefits of a larger dose and faster infusion times. It is likely that manufacturers will move towards generally higher concentrations of product in order to improve the potential for higher dosing and more rapid infusion times which will improve the quality of treatment for patients.

\section{Treatment protocols}

Once a diagnosis has been made, this should be fully explained to the patient and their family. The implications of this lifelong diagnosis and potential complications should be outlined at an early stage. Therapeutic options should then be discussed with the patient, and appropriate written information given to allow them to make an informed choice.

Many patients receive immunoglobulin therapy via the intravenous route, most commonly in the hospital or clinic setting but, in some countries, in the home setting after appropriate training. Replacement dosing has historically been in the range of $200-600 \mathrm{mg} / \mathrm{kg}$ body weight, given at an interval of 2-4 weeks. Routine cannulation is all that is required, and infusions generally last a few hours, depending on the manufacturer's guidelines for infusion rates.

\section{Home therapy}

Patients and their carers can be trained to undertake therapy at home. ${ }^{11,12}$ Once patients are stabilized and can tolerate therapy, usually with intravenous immunoglobulin, they should be offered the option of home therapy. Formal psychological evaluations are not routinely performed, but discussions with the patient and carers are held after written information has been given before accepting a patient and carer onto the training program. Willingness to undertake the training and a stable home environment are the key factors to successful home therapy. Many centers in the UK have experience and expertise in training patients to self-administer intravenous immunoglobulin at home. Hospital-based training involves achievement of competence in aseptic technique, intravenous cannulation, preparation of the delivery system, and management of adverse reactions. When patients and carers are felt to be ready to undertake home infusions, a formal agreement is reached between the patient and their carer, the medical and nursing teams, and the general practitioner that home treatment can commence. The nursing team makes a home visit for the first infusion to confirm competence in the home setting. Many patients in the UK and other European countries successfully undertake this form of treatment. 
Training for home subcutaneous immunoglobulin therapy is technically much more straightforward than for intravenous immunoglobulin therapy. The insertion of a butterfly needle or equivalent using standard aseptic techniques can be readily shown to patients or carers for children, and the use of lowvolume, battery-operated pump devices allows infusions of up to $20 \mathrm{~mL}$ at a single site to be delivered in times of around one hour. There are several pump designs, including battery-free devices such as the Freedom 60, a clockwork device that also allows simultaneous infusions into a number of sites. Larger children and adults usually need to infuse at two sites either on the anterior abdomen or thigh. There is some evidence that a rapid push technique is acceptable to patients. ${ }^{13}$

The use of subcutaneous immunoglobulin offers a number of significant practical advantages to patients and their carers, and can be used for home treatment where intravenous immunoglobulin is not practical in the home environment. Most UK and many European and US centers have taken full advantage of this, and have active home training programs for children and increasingly for adults as well.

\section{Efficacy of immunoglobulin therapy}

There are large numbers of randomized controlled trials examining the effectiveness of immunoglobulin treatment, but no placebo-controlled trial data are available. The UK Medical Research Council reported 201 case histories of immunodeficient patients treated with low doses $(0.1 \mathrm{~g} / \mathrm{kg} / \mathrm{month})$ of intramuscular immunoglobulin, with a 10 -year survival rate of $37 \%{ }^{14}$ In contrast, Cunningham-Rundles and Bodian in $1993^{3}$ reported 248 patients with common variable immunodeficiencies, the vast majority of whom received intravenous immunoglobulin $0.4 \mathrm{~g} / \mathrm{kg} / \mathrm{month}$ throughout the period of observation, and these patients had a 10 -year survival of $78 \%$. Liu et a ${ }^{15}$ concluded from these data that mortality was markedly higher in those treated with low-dose immunoglobulin. More recent European data show a further increase in survival rates. ${ }^{16}$ However, factors in addition to increased immunoglobulin dose, such as improved diagnosis and management of complications, are likely to have made a significant contribution to the increased survival seen in patients treated with intravenous immunoglobulin.

Replacement immunoglobulin therapy reduces the rate of bacterial infection, days of antibiotic usage, days of fever, hospital admissions, and incidence of pneumonia. ${ }^{17-20}$ Patients receiving higher-dose therapy have significantly fewer total episodes of infections per patient, and the duration of infection is significantly shorter than with low-dose therapy. ${ }^{21}$
Previous studies comparing intramuscular immunoglobulin at $0.08-0.1 \mathrm{~g} / \mathrm{kg} /$ month with intravenous immunoglobulin at $0.4 \mathrm{~g} / \mathrm{kg} / \mathrm{month}$ also showed that higher doses of immunoglobulin reduce bacterial infection rates. ${ }^{22}$

Low levels of $\operatorname{IgG}(<6 \mathrm{~g} / \mathrm{L})$ immediately pretherapy (termed trough levels) are associated with moderate lung damage (bronchiectasis). ${ }^{23}$ Significantly higher rates of pneumonia (per patient-year) were found in patients with trough IgG levels $<5 \mathrm{~g} / \mathrm{L}(P=0.06) .{ }^{23}$ There was also an increased risk of chronic lung disease and sinusitis with time in patients with low IgG levels. ${ }^{4}$ Data from Roifman et al in $2003{ }^{17}$ showed that trough IgG levels $>9 \mathrm{~g} / \mathrm{L}$ reduced validated infection rates from more than $10 \%$ to $5.6 \%$. It has been shown that there is a significant correlation between prevention of pneumonia and trough $\operatorname{IgG}$ levels $(P=0.012) .{ }^{18}$ More recently, a large multicenter study demonstrated that intravenous immunoglobulin therapy should be aimed at maintaining a trough level $>4 \mathrm{~g} / \mathrm{L}$ to retain a reduced incidence of pneumonia post-therapy, and that patients with common variable immunodeficiency who have low IgA $(<0.07 \mathrm{~g} / \mathrm{L}), \mathrm{IgM}$, and bronchiectasis at presentation have a higher risk of pneumonia despite therapy. ${ }^{24}$

A crossover study comparing intravenous and subcutaneous immunoglobulin therapy demonstrated equivalent efficacy in terms of infection frequency, ${ }^{25}$ and this has been demonstrated more recently in further studies. ${ }^{26,27}$ However, dosing when changing patients from intravenous to subcutaneous treatment should be adjusted on an individual basis to achieve similar levels of $\operatorname{IgG}^{28}$

These data support the contention that, in general, higher doses of immunoglobulin and higher trough levels are associated with fewer infections. However, it is important to note that this does not translate into ideal dosage or trough IgG levels for all patients, because the level at which infections are prevented varies widely between patients. Recent data suggest that clinical measurements in individual patients may be more important than aiming for a specific trough IgG level, ${ }^{29}$ although a meta-analysis concluded that progressively higher trough IgG levels (achieved by increased dosing) correlated with a reduced incidence of pneumonia during therapy. ${ }^{30}$ It is also unclear if a starting dose adjusted for body weight is an appropriate approach for all patients There was no relationship between annual dose and trough IgG level, regardless of infusion frequency, or adjustment for weight or body mass index. ${ }^{31}$ Whether or not increased doses are truly associated with an improved outcome has yet to be established. Despite this lack of clear evidence, surveys suggest many clinicians use dosing to achieve appropriate trough 
levels, ${ }^{32}$ and guidelines attempting to standardize treatment dosing and frequency have been produced by a number of different countries, often as part of demand management strategies. $^{33,34}$

\section{Risks of immunoglobulin therapy}

The risks of immunoglobulin therapy fall into three main categories. Immediate reactions can occur during infusions which can be severe, including anaphylaxis. Patients may experience a clinical syndrome similar to type I hypersensitivity reactions, with urticaria, angioedema, bronchospasm, and, potentially, cardiovascular collapse. The etiology of such reactions remains unclear, although there have been reports of $\operatorname{IgE}$ antibodies potentially directed against $\operatorname{IgA}$ found in the infusion product. In addition, there are reports of the presence of $\mathrm{IgG}$ antibodies directed against IgA, as reviewed previously, ${ }^{35}$ although the area remains controversial. As noted above, manufacturers attempt to keep the IgA content of their products as low as possible to minimize this risk. Of probably greater risk is the presence of subacute bacterial or low-grade viral infection and the incidence of potentially severe side effects when the patient is acutely infected remains high. For this reason, clinical units commonly operate a policy of ensuring that the patient is apyrexial for a 48-hour period prior to any infusion.

Patients can also experience delayed reactions to immunoglobulin products, occurring several hours after infusion, and most commonly involving headache, backache, and occasional rigors. Again, the precise etiology of these reactions remains unclear, although both immune complex formation and reactivity to components used in the manufacturing process which appear in the final product are thought to be implicated in such reactions. Some reactions may relate to fast infusion times. The osmotic activity of the intravenous immunoglobulin preparations, particularly in older products where carbohydrate moieties are used as stabilizers, may cause a degree of fluid shift into the intravascular space during infusion. This may be exacerbated if the patient is relatively dehydrated, and may contribute to headache and other potentially delayed side effects that are seen. It is likely that residual infections, particularly in patients who have endorgan damage such as bronchiectasis or sinusitis, may well be important in the nature of these reactions. It is clear that patients tolerate different products to differing extents, ${ }^{36}$ and if reactions persist, the brand of intravenous immunoglobulin should be changed. Generally, however, there is general acceptance that the patient should be maintained on the same product if at all possible to reduce the risk of transmission of infection, although there is no direct evidence to support this practice.

Adverse reactions to subcutaneous immunoglobulin appear to be generally much less common. This seems more likely to be as a consequence of the route of administration, with subcutaneous therapy being delivered into a relatively inert space when compared with the intravenous route, rather than inherent differences between intravenous and subcutaneous products.

There is an inevitable risk from pooled donated plasma of the transmission of plasma-borne infectious agents. Intravenous immunoglobulin produced by cold-ethanol fractionation has been regarded as inherently safer than those products prepared from cryoprecipitate fractions, such as factor VIII. However, transmission of hepatitis C infection has been reported previously, although not recently. These outbreaks have resulted in a high level of vigilance regarding donor and plasma selection, and a review of manufacturing processes to assess their impact on viral reduction or inactivation. Cold-ethanol fractionation and incubation at low $\mathrm{pH}$ both contribute to significant reduction in viral concentrations under experimental conditions, although these are not regarded as sufficient. Additional measures, including pasteurization, solvent detergent treatment, caprylic acid treatment, and nanofiltration, have all been demonstrated to remove viral particles to a significant extent during the manufacturing process. Manufacturers currently use a multistage approach with all of the above methods, often used in combination.

Historically, the risk of transmission of viral infection has been of major concern, with previous outbreaks of hepatitis $\mathrm{C}$ infection from contaminated immunoglobulin batches being reported in the late 1980s. It is likely that donor screening, plasma quarantine, and additional viral inactivation steps will reduce this risk substantially. Infusion-related risks of adverse reaction have been reduced considerably in recent years, due to improved manufacturing processes (see above). Viral transmission has not been reported since the last outbreak of hepatitis $\mathrm{C}$ nearly 20 years ago. ${ }^{37,38}$

The other major long-term risk, albeit at this stage theoretical, is the transmission of prions in plasma. The epidemic of bovine spongiform encephalopathy in the UK and a small number of other countries in the 1990s, as well as the reported link between bovine spongiform encephalopathy and a novel form of Creutzfeld-Jacob disease lead to major concerns about the safety of plasma donated from individuals resident in those countries affected by bovine spongiform encephalopathy, in particular the UK. As a result of these concerns, 
a ban was placed upon the use of UK-derived plasma for the manufacturer of immunoglobulin and other plasma-derived products. This ban remains in place and is likely to do so for the foreseeable future. Manufacturers' own assessments indicate that significant removal of prion particles (4-5 log reduction) occurs during the fractionation process, filtration steps, and precipitation procedures, suggesting that the risk transmission from immunoglobulin products is likely to be extremely low. However, case reports of transmission of new variant Creutzfeld-Jacob disease by blood transfusion indicate that a theoretical risk remains, and it is critical that, during the consenting process, patients, their families and carers, are made fully aware of the long-term potential risk from treatment. Clearly, these risks must be balanced against the evidence of benefit from therapy, outlined below.

\section{Consequences of delayed therapy}

Data from the UK in the later 1980s and early 1990s indicated that patients with primary antibody deficiencies experienced a median diagnostic delay of 3.5 years. ${ }^{39,40}$ More recent data suggest that this may have declined to a median of one year. ${ }^{41}$ In the UK, this reduction in delay may have been attributable to the publication of previous reports, including the UK Consensus Document in $1994^{42}$ and the distribution of national guidelines in 1995. However, the delay in diagnosis depends on the type of antibody deficiency, and for some conditions (eg, IgG subclass deficiency), the average delay was found to be in excess of 10 years, ${ }^{41}$ although it is now widely accepted that IgG subclass deficiency in isolation is not a disorder in which immunoglobulin replacement therapy is required.

Diagnostic delay results in treatment delay and morbidity following further infections. An episode of pneumonia before treatment results in a 10-fold increase in risk of pneumonia after therapy. ${ }^{19}$ Inadequate replacement therapy with immunoglobulin places the patient at greater risk of recurrent respiratory tract infections, chronic bronchitis, and rhinosinusitis. ${ }^{2,3}$ Infectious diarrhea occurs with increased frequency in untreated patients with both common variable immunodeficiency and X-linked agammaglobulinemia. ${ }^{43}$ Central nervous system infections have been reported both as a presenting feature and complication of primary antibody deficiency, particularly prediagnosis or during suboptimal therapy. ${ }^{23}$ Such infectious episodes are significantly reduced with adequate immunoglobulin replacement therapy.

In addition to acute infection, end-organ damage can result from delayed diagnosis and therapy. End-stage lung disease with the development of respiratory insufficiency remained the commonest cause of morbidity in large cohort studies from the 1990s, ${ }^{2,3}$ although there is a lack of more recent data. Patients may also suffer from autoimmune hematological disorders. In the largest case series of common variable immunodeficiency reported (326 patients), ${ }^{44} 11 \%$ had a history of autoimmune hematological disease.

Rheumatological complications of primary antibody deficiencies are primarily those due to acute or chronic infection that resolve on appropriate antibiotic therapy and are prevented by the institution of immunoglobulin therapy. ${ }^{45}$ Skin infections may be fungal, bacterial, or viral. ${ }^{46}$ Patients with primary antibody deficiencies on immunoglobulin therapy remain at risk of a number of organ-specific and systemic complications, including inflammatory bowel disease, neurodegeneration, and malignancy. ${ }^{47}$ Estimates of the overall increased risk of malignancy vary from 1.8-fold to 13 -fold, with the risk linked to the primary antibody deficiency rather than the genetic background of the individual. ${ }^{48}$ The occurrence of these and other complications emphasizes the need for regular clinical review by appropriately trained specialists and further investigations as required.

\section{Current availability}

There are a number of plasma fractionation companies manufacturing both intravenous and subcutaneous products. Their current availability in the UK is shown in Table 1. A majority of manufacturers provide both intravenous and subcutaneous products derived from the same plasma donation pool, and as outlined above, the current trend is towards higher concentration of product, with the benefits of reduced volume and infusion time.

It is clear that patients with primary immunodeficiencies tolerate certain products more than others. This may partly be due to the IgA content of products and the presence of anti-IgA antibodies in the patient, although the data on this issue remain unclear and, in any event,

Table IA Intravenous immunoglobulin products currently available in the UK

\begin{tabular}{lll}
\hline Product & Manufacturer & Concentration (\% v/v) \\
\hline Kiovig $^{\circledR}$ & Baxter & 10 \\
Vigam $^{\circledR}$ & BPL & 5 \\
Gammaplex $^{\circledR}$ & BPL & 5 \\
Intratect $^{\circledR}$ & Biotest & 10 \\
Privigen $_{\text {Flebogamma }}{ }^{\circledR}$ DIF 5\% & Grifols & 5 \\
Flebogamma $^{\circledR}$ DIF 10\% & Grifols & 10 \\
Octagam $^{\circledR} 5 \%$ & Octapharma & 5 \\
Octagam $^{\circledR 10 \%}$ & Octapharma & 10 \\
\hline
\end{tabular}


Table IB Subcutanous immunoglobulin products currently available in the UK

\begin{tabular}{lll}
\hline Product & Manufacturer & Concentration (\% v/v) \\
\hline Subcuvia $^{\circledR}$ & Baxter & 16 \\
Subgam $^{\circledR}$ & BPL & 16 \\
Vivaglobin $^{\circledR}$ & CSL Behring & 16 \\
Hizentra $^{\circledR}$ & CSL Behring & 20 \\
\hline
\end{tabular}

manufacturers all ensure that the IgA content of their products is as low as possible. However, the issue of variable tolerability underlies the consensus best practice approach of ensuring that patients have access to a range of products, and this is endorsed by national professional societies, eg, The UK Primary Immunodeficiency Network (UK PIN, www.ukpin.org.uk). In addition, it is desirable to ensure continuity of product in an individual patient unless a change of product is required clinically. Patients should not have their product changed purely on the grounds of cost or convenience.

\section{Quality of life issues}

There is only one study that has directly compared healthrelated quality of life before and after immunoglobulin therapy. ${ }^{49}$ This study of 25 patients with common variable immunodeficiency or X-linked agammaglobulinemia used analysis of medical records, data registers, and questionnaires (a study-specific questionnaire in addition to the
Sickness Impact Profile ${ }^{50}$ ) to assess the overall quality of life prior to and 18 months after initiation of subcutaneous immunoglobulin therapy. After 18 months of subcutaneous immunoglobulin therapy, patients reported significant improvements across all areas of health-related function to levels comparable with those of healthy individuals. In addition, the patient group reported reduced fear of infections and decreased anxiety about their future health. It is reasonable to conclude that initiation of immunoglobulin therapy was a major factor in the improvements seen, although other factors relating to diagnosis and support from health professionals are likely to have been contributory. Other studies support the overall concept that patients prefer home-based therapy and report improved quality of life when transferring from hospital-based intravenous immunoglobulin therapy. ${ }^{27,51}$

Other studies have assessed the quality of life of individuals already receiving immunoglobulin replacement therapy ${ }^{52}$ Eighty-three percent of a cohort of adult patients with X-linked agammaglobulinemia rated their health as good, very good, or excellent. The responses indicated that the adult males with X-linked agammaglobulinemia had quality of life equivalent to that of the general male population, other than in their perception of their own health. In this study, patients with X-linked agammaglobulinemia had a better quality of life in every parameter when compared with individuals with diabetes. Although direct comparison is

Table 2 Clinical benefits of immunoglobulin replacement therapy in primary antibody deficiency

\begin{tabular}{|c|c|}
\hline Issue & Grade of evidence \\
\hline Increased life expectancy & $2++$ \\
\hline Reduction in rate of bacterial infection & $2++$ \\
\hline Greater morbidity from diagnostic and treatment delay & $2++$ \\
\hline Reduction in infections with increased dose & $\mathrm{I}++$ \\
\hline Improved quality of life with replacement therapy & $2++$ \\
\hline \multicolumn{2}{|l|}{ Grade of evidence } \\
\hline $\mathrm{I}++$ & $\begin{array}{l}\text { High quality meta-analyses, systematic reviews of RCTs, or RCTs with a very } \\
\text { low risk of bias }\end{array}$ \\
\hline $1+$ & $\begin{array}{l}\text { Well conducted meta-analyses, systematic reviews of RCTs, or RCTs with a } \\
\text { very low risk of bias }\end{array}$ \\
\hline $\mathrm{I}-$ & Meta-analyses, systematic reviews of RCTs, or RCTs with a high risk of bias \\
\hline \multirow[t]{2}{*}{$2++$} & High quality systematic reviews of case-control or cohort studies \\
\hline & $\begin{array}{l}\text { High quality case-control or cohort studies with a very low risk of confounding } \\
\text { bias, or chance and a high probability that the relationship is causal }\end{array}$ \\
\hline $2+$ & $\begin{array}{l}\text { Well conducted case-control or cohort studies with a very low risk of } \\
\text { confounding bias, or chance and a moderate probability that the relationship } \\
\text { is causal }\end{array}$ \\
\hline $2-$ & $\begin{array}{l}\text { Case-control or cohort studies with a high risk of confounding bias, or chance } \\
\text { and a significant risk that the relationship is not causal }\end{array}$ \\
\hline 3 & Nonanalytic studies, eg, case reports, case series \\
\hline 4 & Expert opinion \\
\hline
\end{tabular}

Abbreviation: RCT, randomized controlled trial. 
not possible, more historical data on patients with X-linked agammaglobulinemia indicate significant morbidity and poor survival rates in adult life, particularly from the era before intravenous immunoglobulin, ${ }^{53}$ indicating that earlier diagnosis and prompt initiation of adequate immunoglobulin replacement therapy improves overall quality of life. This is supported by recent data from Iran. ${ }^{54}$

In contrast, a study comparing patients with common variable immunodeficiency receiving intravenous immunoglobulin with patients suffering from diabetes mellitus or congestive cardiac failure found that patients with common variable immunodeficiency had significantly worse healthrelated quality of life than patients with either of the other conditions, unrelated to socioeconomics or disease-specific factors. ${ }^{55}$ However, this does not indicate that initiation of immunoglobulin replacement therapy fails to improve overall life quality.

In general, studies looking at quality of life issues in primary antibody deficiencies suggest that replacement therapy with immunoglobulin given either by the intravenous or subcutaneous route is a contributory factor in improvement of quality of life. There are no studies assessing how diagnostic or treatment delay affects this, although pretreatment data provide some indication as to the overall poor quality of life suffered by individuals with either undiagnosed or untreated primary antibody deficiencies. It could be inferred that because an increased dose reduces the overall infection burden, that this would improve quality of life further, but this has not been demonstrated directly.

\section{Economic benefits of diagnosis and appropriate therapy}

There are a small number of studies assessing the cost-effectiveness of immunoglobulin therapy, but none comparing no therapy with replacement therapy. All studies are limited by the costs of immunoglobulin at the time of the study. However, an early study compared the potential cost-effectiveness of changing from intramuscular to intravenous immunoglobulin. ${ }^{56}$ Because intramuscular immunoglobulin therapy has often been used as a surrogate marker for no effective therapy, this study represents an attempt to compare therapy with no therapy. Twenty-three children with a variety of primary antibody deficiency states were assessed in the 2 years prior to and in the 3 years following the change from intramuscular immunoglobulin to intravenous immunoglobulin. In particular, the number of days with antibiotics, number of absences from school, number of days in bed or hospital, and the number of days with infection or fever were assessed at 3-weekly intervals. The change from intramuscular immunoglobulin to intravenous immunoglobulin produced a $90 \%$ fall in the number of hospital bed days. Based on 1990 Italian costs, the relative costs of antibiotic therapy, hospitalization, and immunoglobulin drug costs per month for a $20 \mathrm{~kg}$ child were 953,000 Lira for intramuscular immunoglobulin and 826,000 Lira for intravenous immunoglobulin. Given that the quoted figures for drug costs were 160,000 Lira for intramuscular immunoglobulin and 468,000 Lira for intravenous immunoglobulin, this illustrates the significant overall cost-saving of adequate therapy using intravenous immunoglobulin.

Other studies have compared the estimated relative overall treatment costs of intravenous immunoglobulin with those of subcutaneous immunoglobulin. Such studies are limited by the relative drug costs, which in the case of the study by Gardulf et al in $1995^{57}$ were based on an approximate cost of $\$ 14,000$ per annum for intravenous immunoglobulin against $\$ 4650$ for subcutaneous immunoglobulin. Perhaps not surprisingly, this study reported a lower overall cost for home subcutaneous immunoglobulin therapy compared with hospital-based intravenous immunoglobulin therapy. A more recent study ${ }^{58}$ compared the costs with the German health insurance system of providing intravenous immunoglobulin or subcutaneous immunoglobulin from 18 centers in Germany. Costs were taken from the standard tariffs for drugs and health services in the German health care system in 2003. The costs considered were for the immunoglobulin products, the materials required for infusion, medical treatments, and sick leave (for caregivers of children). For adults, the overall costs per annum were 31,027 Euro for intravenous immunoglobulin and 14,893 Euro for subcutaneous immunoglobulin. Within these costs, the drug costs were 30,456 Euro and 13,874 Euro, respectively. Considering that these data are derived from a unit cost of 86.40 Euro for intravenous immunoglobulin and 38.54 Euro for subcutaneous immunoglobulin, at price equivalence there is effectively no cost difference between the two therapy routes. Smaller but similar costs applied to children in the study.

As part of a cost-effectiveness review of immunoglobulin therapy, Hyde et al developed a Markov model to assess the cost-effectiveness of immunoglobulin therapy. This used derived mortality data based on survival estimates for 
current immunoglobulin replacement regimes (mainly intravenous immunoglobulin) compared with historical data (mainly intramuscular immunoglobulin), although the authors acknowledged that such a comparison was difficult due to the lack of placebo-controlled randomized trial data. ${ }^{15}$ Nevertheless, a conversion of the costs used by Hogy et $\mathrm{al}^{58}$ into UK costs resulted in estimated costs per annum of $£ 18,600$ for hospital-based intravenous immunoglobulin therapy and $£ 11,580$ for home-based intravenous immunoglobulin therapy. Costs of subcutaneous immunoglobulin therapy (exclusively based at home) were $£ 11,760$, reflecting the similar unit costs of subcutaneous immunoglobulin and intravenous immunoglobulin in the UK. Taking these data assumptions into account, the economic model predicted a base-case incremental cost-effectiveness ratio per quality-adjusted life-year of approximately $£ 30,000$. The incremental cost-effectiveness ratio for immunoglobulin therapy in primary immunodeficiency compares favorably with the use of prophylactic therapy in hemophilia. ${ }^{59}$

\section{Conclusion}

Immunoglobulin replacement therapy has been used for over 50 years in the treatment of primary immunodeficiencies, with advances in manufacturing processes allowing safe and efficient delivery of intravenous immunoglobulin for the past 30 years. This has transformed the management of patients with primary antibody deficiencies, resulting in prolonged survival, reduced morbidity, and improved quality of life (Table 2). The development of home therapy programs with both intravenous and subcutaneous immunoglobulin has led to further improvements in quality of life for this patient group, and may compare favorably with hospital-based treatment programs in terms of cost-effectiveness. There is clear evidence that delayed initiation of immunoglobulin replacement therapy results in increased morbidity and mortality, and improvements in overall survival seen more recently are most likely to relate to the earlier recognition, referral, and treatment of patients. Risks of immunoglobulin therapy relate to the potential for plasma-borne infections, although modern manufacturing processes have reduced this to a highly acceptable level. Risks of prion transmission remain a concern although, as yet, there is no direct evidence that this can occur. Adverse reactions to immunoglobulin are often predictable, and can be effectively managed by patient education and appropriate planning. Available evidence indicates that immunoglobulin replacement therapy is cost-effective within current assessments of health care delivery.

\section{Disclosure}

The author reports no conflict of interest in this work.

\section{References}

1. Notarangelo LD, Fischer A, Geha RS, et al. Primary immunodeficiencies: 2009 update. J Allergy Clin Immunol. 2009;124(6):1161-1178.

2. Hermaszewski RA, Webster AD. Primary hypogammaglobulinaemia: a survey of clinical manifestations and complications. $Q J \mathrm{Med}$. 1993;86(1):31-42.

3. Cunningham-Rundles C, Bodian C. Common variable immunodeficiency: clinical and immunological features of 248 patients. Clin Immunol. 1999;92(1):34-48.

4. Kainulainen L, Varpula M, Liippo K, Svedstrom E, Nikoskelainen J, Ruuskanen O. Pulmonary abnormalities in patients with primary hypogammaglobulinemia. J Allergy Clin Immunol. 1999;104(5):1031-1036.

5. Michel M, Chanet V, Galicier L, et al. Autoimmune thrombocytopenic purpura and common variable immunodeficiency: analysis of 21 cases and review of the literature. Medicine (Baltimore). 2004;83(4):254-263.

6. Bruton OC. Agammaglobulinemia (congenital absence of gamma globulin); report of a case. Med Ann Dist Columbia. 1953;22(12): 648-650.

7. Church JA, Leibl H, Stein MR, et al. Efficacy, safety and tolerability of a new $10 \%$ liquid intravenous immune globulin [IGIV $10 \%$ in patients with primary immunodeficiency. J Clin Immunol. 2006;26(4):388-395.

8. Shah SR. A newer immunoglobulin intravenous (IGIV) - Gammagard liquid 10\%: evaluation of efficacy, safety, tolerability and impact on patient care. Expert Opin Biol Ther. 2008;8(6):799-804.

9. Stein MR, Nelson RP, Church JA, et al. Safety and efficacy of Privigen, a novel $10 \%$ liquid immunoglobulin preparation for intravenous use, in patients with primary immunodeficiencies. J Clin Immunol. 2009;29(1):137-144.

10. Berger M, Pinciaro PJ, Althaus A, et al. Efficacy, pharmacokinetics, safety, and tolerability of Flebogamma $10 \%$ DIF, a high-purity human intravenous immunoglobulin, in primary immunodeficiency. J Clin Immunol. 2010;30(2):321-329.

11. Gardulf A, Hammarstrom L, Smith CI. Home treatment of hypogammaglobulinaemia with subcutaneous gammaglobulin by rapid infusion. Lancet. 1991;338(8760):162-166.

12. Chapel H, Brennan V, Delson E. Immunoglobulin replacement therapy by self-infusion at home. Clin Exp Immunol. 1988;73(1):160-162.

13. Shapiro R. Subcutaneous immunoglobulin therapy by rapid push is preferred to infusion by pump: a retrospective analysis. J Clin Immunol. 2010;30(2):301-307.

14. UK Medical Research Council. Hypogammaglobulinaemia in the United Kingdom. Special Report Series London HMSO. London, UK: UK Medical Research Council; 1971.

15. Liu Z, Albon E, Hyde C. The effectiveness and cost-effectiveness of immunoglobulin replacement therapy for primary immunodeficiency and chronic lymphocytic leukaemia: a systematic review and economic evaluation. West Midlands Health Technology Assessment Group, Dept of Public Health and Epidemiology, University of Birmingham, 2006.

16. Chapel H, Lucas M, Lee M, et al. Common variable immunodeficiency disorders: division into distinct clinical phenotypes. Blood. 2008; 112(2):277-286

17. Roifman CM, Schroeder H, Berger M, et al. Comparison of the efficacy of IGIV-C, 10\% (caprylate/chromatography) and IGIV-SD, 10\% as replacement therapy in primary immune deficiency. A randomized double-blind trial. Int Immunopharmacol. 2003;3(9):1325-1333.

18. Aghamohammadi A, Moin M, Farhoudi A, et al. Efficacy of intravenous immunoglobulin on the prevention of pneumonia in patients with agammaglobulinemia. FEMS Immunol Med Microbiol. 2004;40(2): 113-118. 
19. Busse PJ, Razvi S, Cunningham-Rundles C. Efficacy of intravenous immunoglobulin in the prevention of pneumonia in patients with common variable immunodeficiency. J Allergy Clin Immunol. 2002; 109(6):1001-1004.

20. de Gracia J, Vendrell M, Alvarez A, et al. Immunoglobulin therapy to control lung damage in patients with common variable immunodeficiency. Int Immunopharmacol. 2004;4(6):745-753.

21. Eijkhout HW, van Der Meer JW, Kallenberg CG, et al. The effect of two different dosages of intravenous immunoglobulin on the incidence of recurrent infections in patients with primary hypogammaglobulinemia. A randomized, double-blind, multicenter crossover trial. Ann Intern Med. 2001;135(3):165-174.

22. Cunningham-Rundles C, Siegal FP, Smithwick EM, et al. Efficacy of intravenous immunoglobulin in primary humoral immunodeficiency disease. Ann Intern Med. 1984;101(4):435-439.

23. Plebani A, Soresina A, Rondelli R, et al. Clinical, immunological, and molecular analysis in a large cohort of patients with X-linked agammaglobulinemia: an Italian multicenter study. Clin Immunol. 2002;104(3):221-230.

24. Quinti I, Soresina A, Guerra A, et al. Effectiveness of immunoglobulin replacement therapy on clinical outcome in patients with primary antibody deficiencies: results from a multicenter prospective cohort study. J Clin Immunol. 2011;31(3):315-322.

25. Chapel HM, Spickett GP, Ericson D, Engl W, Eibl MM, Bjorkander J. The comparison of the efficacy and safety of intravenous versus subcutaneous immunoglobulin replacement therapy. $J$ Clin Immunol. 2000;20(2):94-100.

26. Borte M, Bernatowska E, Ochs HD, Roifman CM. Efficacy and safety of home-based subcutaneous immunoglobulin replacement therapy in paediatric patients with primary immunodeficiencies. Clin Exp Immunol. 2011;164(3):357-364.

27. Hoffmann F, Grimbacher B, Thiel J, Peter HH, Belohradsky BH. Home-based subcutaneous immunoglobulin G replacement therapy under real-life conditions in children and adults with antibody deficiency. Eur J Med Res. 2010;15(6):238-245.

28. Berger M, Rojavin M, Kiessling P, Zenker O. Pharmacokinetics of subcutaneous immunoglobulin and their use in dosing of replacement therapy in patients with primary immunodeficiencies. Clin Immunol. 2011;139(2):133-141.

29. Lucas M, Lee M, Lortan J, Lopez-Granados E, Misbah S, Chapel H. Infection outcomes in patients with common variable immunodeficiency disorders: relationship to immunoglobulin therapy over 22 years. J Allergy Clin Immunol. 2010;125(6):1354-1360, e1354.

30. Orange JS, Grossman WJ, Navickis RJ, Wilkes MM. Impact of trough IgG on pneumonia incidence in primary immunodeficiency: A metaanalysis of clinical studies. Clin Immunol. 2010;137(1):21-30.

31. Khan S, Grimbacher B, Boecking C, et al. Serum trough IgG level and annual intravenous immunoglobulin dose are not related to body size in patients on regular replacement therapy. Drug Metab Lett. 2011;5(2):132-136.

32. Yong PL, Boyle J, Ballow M, et al. Use of intravenous immunoglobulin and adjunctive therapies in the treatment of primary immunodeficiencies: A working group report of and study by the Primary Immunodeficiency Committee of the American Academy of Allergy Asthma and Immunology. Clin Immunol. 2010;135(2):255-263.

33. Roifman CM, Berger M, Notarangelo LD. Management of primary antibody deficiency with replacement therapy: summary of guidelines. Immunol Allergy Clin North Am. 2008;28(4):875-876.

34. Shehata N, Palda V, Bowen T, et al. The use of immunoglobulin therapy for patients with primary immune deficiency: an evidence-based practice guideline. Transfus Med Rev. 2010;24 Suppl 1:S28-S50.

35. Rachid R, Castells M, Cunningham-Rundles C, Bonilla FA. Association of anti-IgA antibodies with adverse reactions to gamma-globulin infusion. J Allergy Clin Immunol. 2011;128(1):228-230, e221.

36. Feldmeyer L, Benden C, Haile SR, et al. Not all intravenous immunoglobulin preparations are equally well tolerated. Acta Derm Venereol. 2010;90(5):494-497.
37. Chapel HM, Christie JM, Peach V, Chapman RW. Five-year follow-up of patients with primary antibody deficiencies following an outbreak of acute hepatitis C. Clin Immunol. 2001;99(3):320-324.

38. Quinti I, Pierdominici M, Marziali M, et al. European surveillance of immunoglobulin safety - results of initial survey of 1243 patients with primary immunodeficiencies in 16 countries. Clin Immunol. 2002;104(3):231-236.

39. Spickett GP, Askew T, Chapel HM. Management of primary antibody deficiency by consultant immunologists in the United Kingdom: a paradigm for other rare diseases. Qual Health Care. 1995;4(4):263-268.

40. Blore J, Haeney MR. Primary antibody deficiency and diagnostic delay. Br Med J. 1989;298(6672):516-517.

41. Seymour B, Miles J, Haeney M. Primary antibody deficiency and diagnostic delay. J Clin Pathol. 2005;58(5):546-547.

42. Chapel HM. Consensus on diagnosis and management of primary antibody deficiencies. Consensus Panel for the Diagnosis and Management of Primary Antibody Deficiencies. Br Med J. 1994;308(6928): $581-585$.

43. Kainulainen L, Nikoskelainen J, Ruuskanen O. Diagnostic findings in 95 Finnish patients with common variable immunodeficiency. J Clin Immunol. 2001;21(2):145-149.

44. Wang J, Cunningham-Rundles C. Treatment and outcome of autoimmune hematologic disease in common variable immunodeficiency (CVID). J Autoimmun. 2005;25(1):57-62.

45. Sordet C, Cantagrel A, Schaeverbeke T, Sibilia J. Bone and joint disease associated with primary immune deficiencies. Joint Bone Spine. 2005;72(6):503-514.

46. Wood P. Primary antibody deficiency syndromes. Ann Clin Biochem. 2009;46(Pt 2):99-108.

47. Wood P, Stanworth S, Burton J, et al. Recognition, clinical diagnosis and management of patients with primary antibody deficiencies: a systematic review. Clin Exp Immunol. 2007;149(3):410-423.

48. Mellemkjaer L, Hammarstrom L, Andersen V, et al. Cancer risk among patients with IgA deficiency or common variable immunodeficiency and their relatives: a combined Danish and Swedish study. Clin Exp Immunol. 2002;130(3):495-500.

49. Gardulf A, Bjorvell H, Gustafson R, Hammarstrom L, Smith CI. The life situations of patients with primary antibody deficiency untreated or treated with subcutaneous gammaglobulin infusions. Clin Exp Immunol. 1993;92(2):200-204.

50. Bergner M, Bobbitt RA, Carter WB, Gilson BS. The Sickness Impact Profile: development and final revision of a health status measure. Med Care. 1981;19(8):787-805.

51. Berger M, Murphy E, Riley P, Bergman GE. Improved quality of life, immunoglobulin $\mathrm{G}$ levels, and infection rates in patients with primary immunodeficiency diseases during self-treatment with subcutaneous immunoglobulin G. South Med J. 2010;103(9):856-863.

52. Howard V, Greene JM, Pahwa S, et al. The health status and quality of life of adults with X-linked agammaglobulinemia. Clin Immunol. 2006;118(2-3):201-208.

53. Lederman HM, Winkelstein JA. X-linked agammaglobulinemia: an analysis of 96 patients. Medicine (Baltimore). 1985;64(3): $145-156$.

54. Aghamohammadi A, Montazeri A, Abolhassani H, et al. Health-related quality of life in primary antibody deficiency. Iran J Allergy Asthma Immunol. 2011;10(1):47-51.

55. Tcheurekdjian H, Palermo T, Hostoffer R. Quality of life in common variable immunodeficiency requiring intravenous immunoglobulin therapy. Ann Allergy Asthma Immunol. 2004;93(2):160-165.

56. Galli E, Barbieri C, Cantani A, Solano A, Longhi MA, Businco L. Treatment with gammaglobulin preparation for intravenous use in children with humoral immunodeficiency: clinical and immunologic follow-up. Ann Allergy. 1990;64(2 Pt 1):147-150.

57. Gardulf A, Andersen V, Bjorkander J, et al. Subcutaneous immunoglobulin replacement in patients with primary antibody deficiencies: safety and costs. Lancet. 1995;345(8946):365-369. 
58. Hogy B, Keinecke HO, Borte M. Pharmacoeconomic evaluation of immunoglobulin treatment in patients with antibody deficiencies from the perspective of the German statutory health insurance. Eur J Health Econ. 2005;6(1):24-29.
59. Lippert B, Berger K, Berntorp E, et al. Cost effectiveness of haemophilia treatment: a cross-national assessment. Blood Coagul Fibrinolysis. 2005;16(7):477-485.

\section{Publish your work in this journal}

Therapeutics and Clinical Risk Management is an international, peerreviewed journal of clinical therapeutics and risk management, focusing on concise rapid reporting of clinical studies in all therapeutic areas outcomes, safety, and programs for the effective, safe, and sustained use of medicines. This journal is indexed on PubMed Central, CAS,
EMBase, Scopus and the Elsevier Bibliographic databases. The manuscript management system is completely online and includes a very quick and fair peer-review system, which is all easy to use. Visit http://www.dovepress.com/testimonials.php to read real quotes from published authors.

Submit your manuscript here: http://www.dovepress.com/therapeutics-and-clinical-risk-management-journal 